\title{
Editorial
}

\section{Stochastic Modeling and Financial Applications}

\author{
Ivan Ganchev Ivanov, ${ }^{1}$ Vasile Dragan, ${ }^{2}$ and Oswaldo Luiz do Valle Costa ${ }^{3}$ \\ ${ }^{1}$ Faculty of Economics and Business Administration, Sofia University "St. Kl. Ohridski," 125 Tsarigradsko Shosse Boulevard, \\ bl.3, 1113 Sofia, Bulgaria \\ ${ }^{2}$ Institute of Mathematics "Simion Stoilow" of the Romanian Academy, Calea Grivitei No. 21, P.O. Box 1-764, \\ 014700 Bucharest, Romania \\ ${ }^{3}$ Departamento de Engenharia de Telecomunicações de Controle, Institute Escola Politécnica da Universidade de São Paulo (EPUSP), \\ 05508-900 São Paulo, SP, Brazil
}

Correspondence should be addressed to Ivan Ganchev Ivanov; i_ivanov@feb.uni-sofia.bg

Received 3 December 2013; Accepted 3 December 2013

Copyright (C) 2013 Ivan Ganchev Ivanov et al. This is an open access article distributed under the Creative Commons Attribution License, which permits unrestricted use, distribution, and reproduction in any medium, provided the original work is properly cited.

This special issue brings together several works in the area of advanced modern theory of stochastic modeling and applications in financial and economic fields.

The Basel III banking regulation is a topic addressed in two paper. The paper by M. Mpundu et al. focuses on Basel III and asset securitization. It deals with aspects of the mechanism by which low- and high-quality entities securitize low and high quality assets, respectively, into collateralized debt obligations, under the new Basel III capital and liquidity regulations. The authors develop an illustrative example of low-quality asset securitization for subprime mortgages and present numerical examples to illustrate their key results. The paper by L. N. P. Hlatshwayo et al. focuses on Basel III liquidity risk measures and bank failure. The authors point out that Basel III banking regulation emphasizes the use of liquidity coverage and net stable funding ratios as measures of liquidity risk. The goal of this paper is to approximate these measures by using global liquidity data. In addition the authors compare the risk sensitivity of the aforementioned Basel III liquidity risk measures to those of traditional measures. The authors also use a discrete-time hazard model to study bank failure. One of their conclusions is that Basel III risk measures have limited ability to predict bank failure when compared with their traditional counterparts.

The paper by Kai Chang shows, from empirical evidences, that conditional variance, conditional covariance and their correlation between spot and futures exhibit time-varying trends. Moreover it is claimed that conditional volatility of spot prices, conditional volatility disturbed from futures market, and conditional correlation of market noises implied from spot and futures market have significant effects on dynamic hedge ratios and hedging effectiveness. From this a better hedging efficiency through dynamic hedge ratios with the departures from cost-of-carry theory is obtained.

The paper by Y. Song and L. Lin defines a sub-linear expectation nonlinear regression, having in mind its application on the management and measurement of financial risks. The paper presents a simulation study and a real data analysis to illustrate the new model and methods. The paper by Q. Zhang deals with the terminal-dependent statistical inference for backward stochastic differential equations (BSDE), which arises in financial and ecological modeling.

The paper focuses on the statistical inference for the integral form of forward-backward stochastic differential equations (FBSDE). Simulations are conducted to demonstrate finite sample behaviors of the proposed estimators.

Two papers are not related to financial systems but, instead, are concerned with stochastic modeling and numerical methods. The paper by M. Heydari et al. examines the preemptive stochastic online flowshop with the objective of minimizing the expected makespan. The model assumes that all the jobs arrive overtime, which means that the existence and the parameters of each job are unknown until its release date, the processing time of the jobs is stochastic, and actual processing time is unknown until completion of the job. 
The authors propose a heuristic procedure for this problem, which is applicable whenever the job processing times are characterized by their means and standard deviation. The performance of the proposed heuristic method is explored using some numerical examples.

The paper by J. Ma and Y. Yang deals with hyperchaos numerical simulation and control in a $4 \mathrm{D}$ hyperchaotic system.

The bifurcation diagrams, Lyapunov exponents, hyperchaotic attractors, the power spectrums, and time charts are mapped out through the theory analysis and dynamic simulations. Linear feedback controllers are designed for stabilizing the hyperchaos to the unstable equilibrium points, achieving the goal of a second control which can be more useful in application.

By compiling these papers, we hope to enrich our readers and researchers with respect to these topics related to stochastic modeling and financial applications.

Ivan Ganchev Ivanov

Vasile Dragan

Oswaldo Luiz do Valle Costa 


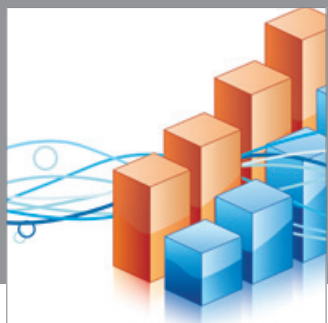

Advances in

Operations Research

mansans

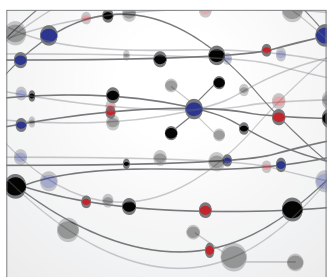

The Scientific World Journal
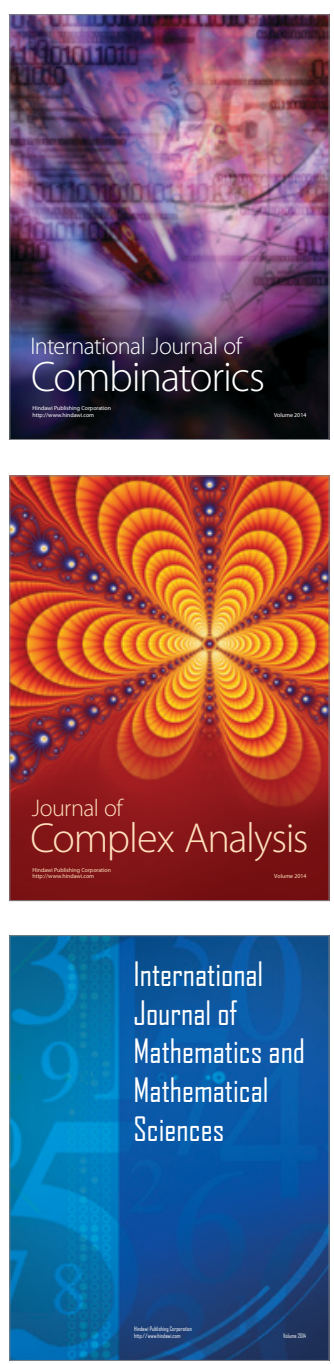
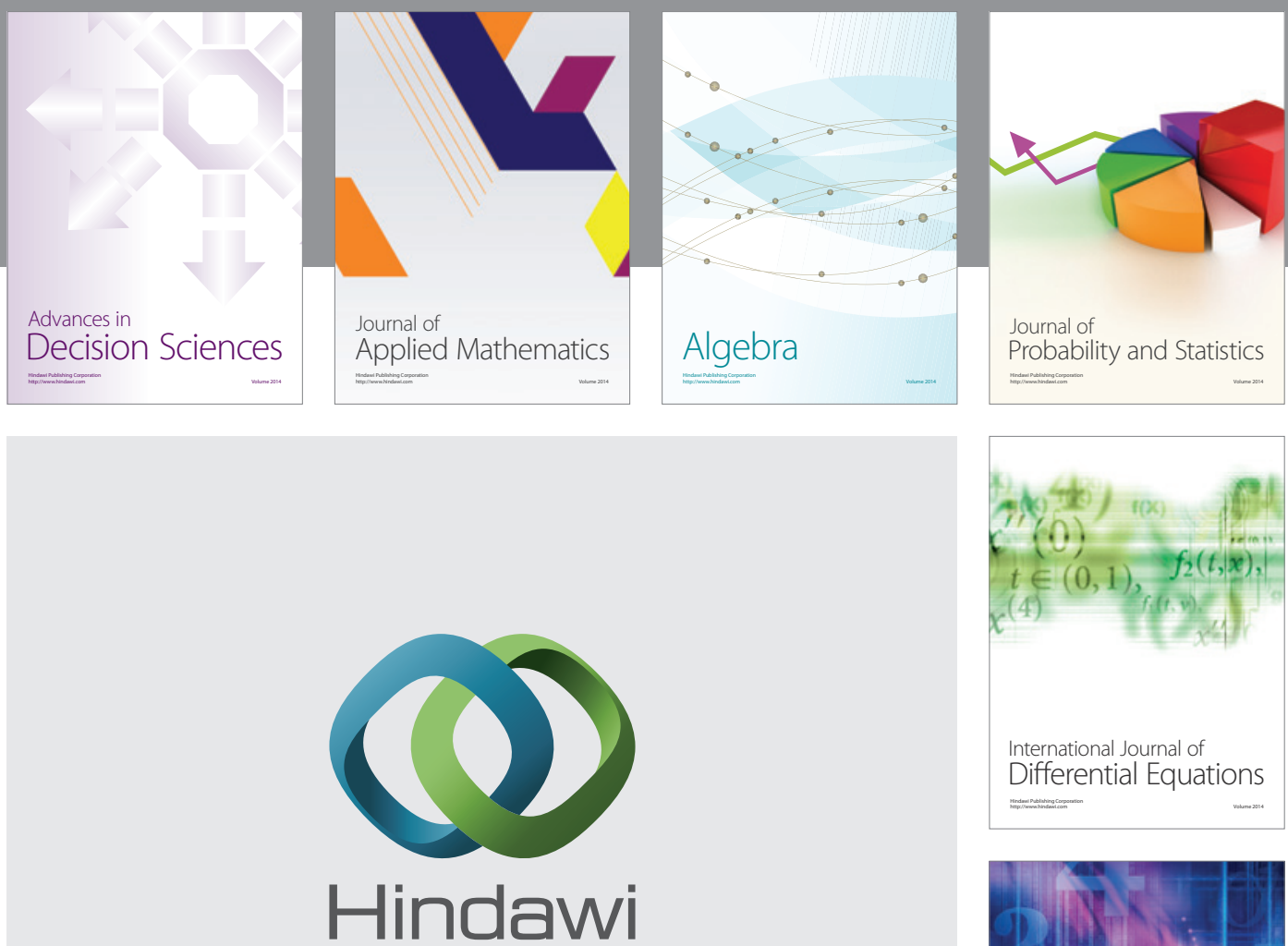

Submit your manuscripts at http://www.hindawi.com
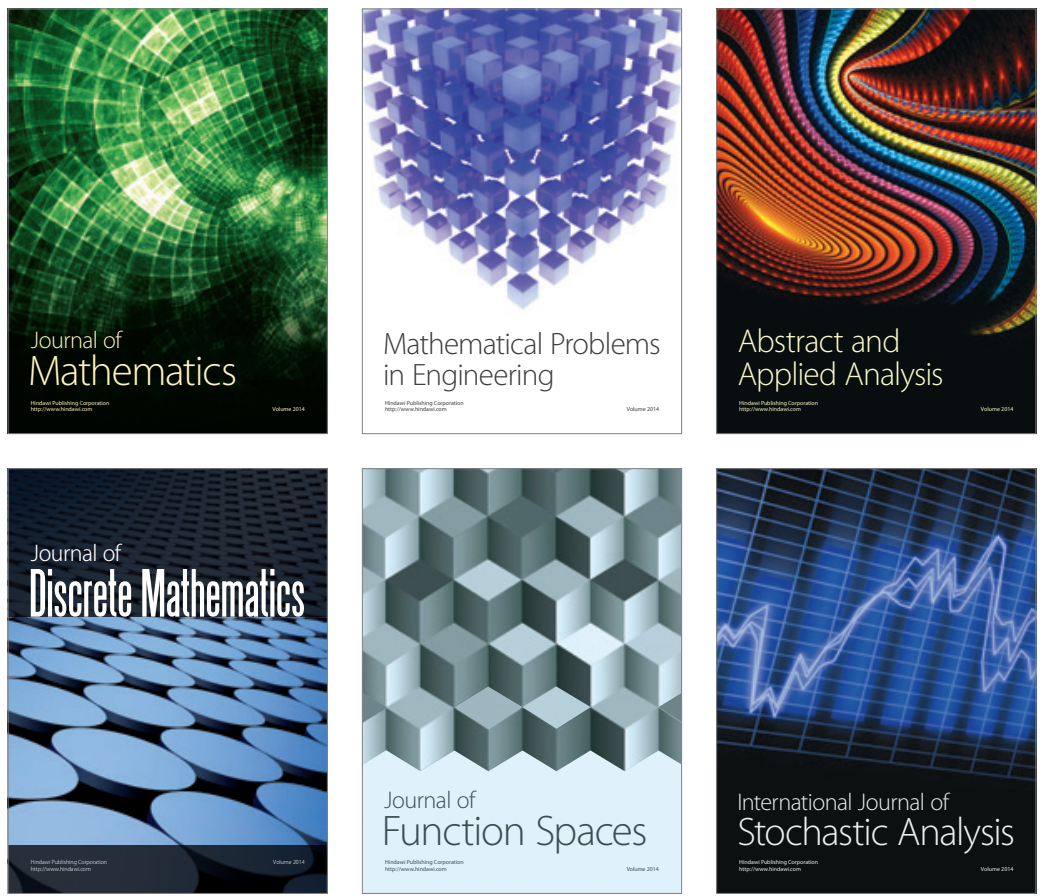

Journal of

Function Spaces

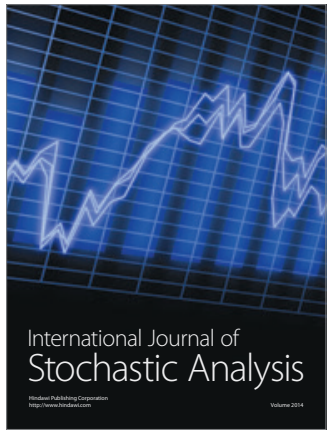

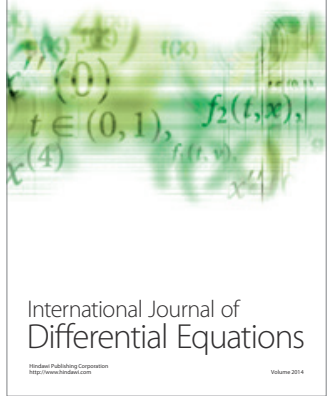
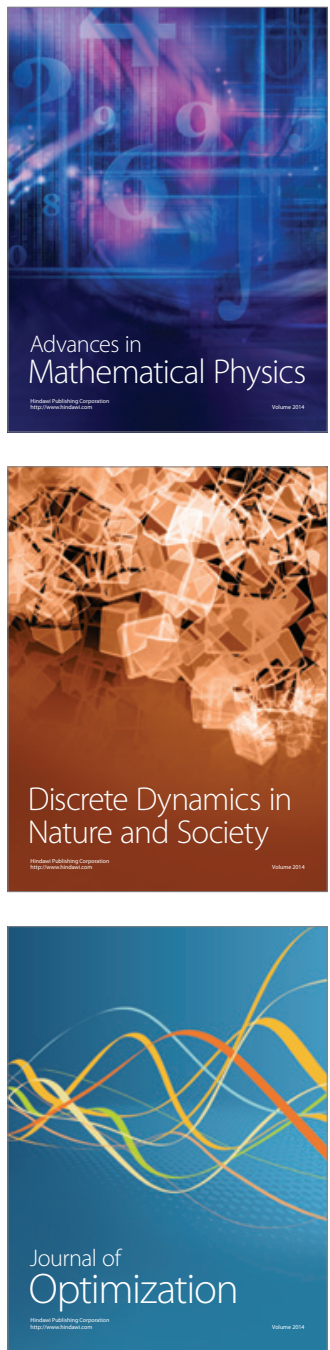